International Journal of Nano Studies \& Technology (IJNST)

\title{
Superparamagnetic Iron Oxide Nanoparticles (SPIONS): The King in The Cosmos of Multimodal \\ Onco-Nanotheranostics.
}

Kanwar J R ${ }^{1^{*}}$, Kamalapuram S K ${ }^{1}$

${ }^{1}$ Nanomedicine-Laboratory of Immunology and Molecular Biomedical Research (LIMBR), School of Medicine (SoM), Faculty of Health, Deakin University, Waurn Ponds, Victoria 3217, Australia.

\section{*Corresponding Author:}

Jagat R Kanwar,

Nanomedicine-Laboratory of Immunology and Molecular Biomedical Research (LIMBR), School of Medicine (SoM), Faculty of Health, Deakin University, Waurn Ponds, Victoria 3217,

Australia.

E-mail: jagat.kanwar@deakin.edu.au

Tel: +613 52271148; Fax: +61352272539

Received: February 15,2013

Published: April 22, 2013

Citation: Kanwar J R. (2013). Superparamagnetic Iron Oxide Nanoparticles (SPIONS): The King in The Cosmos of Multimodal Onco-Nanotheranostics, Int J Nano Stud Technol, 02(01), 01-02. doi: http:// dx.doi.org/10.19070/2167-8685-130003e

Copyright: Kanwar $J R^{\odot} 2013$. This is an open-access article distributed under the terms of the Creative Commons Attribution License, which permits unrestricted use, distribution and reproduction in any medium, provided the original author and source are credited.

There is an extensional necessity for the major amelioration of hybrid multimodal nanotheranostics, that can offer ulterior theranostic possibilities in the field of nano - oncology [1,2]. Novel class of nanotheranostic medicines certainly warrant more throughput therapeutic abilities in comparison with conventional platforms [3]. One such platform that dawned in the era of existing treatment efficacies is superparamagnetic iron oxide nanoparticles (SPIONS) nanotheranostics [4]. SPIONS are approved by food and drug administration, USA (FDA), safe, biocompatible and exhibit astounding capabilities in prominence, in realms of (1) diagnostic and prognostic platforms - nanofluidic lap on chip devices, nanoarray systems, nanosensors [5], (2)targeted multimodal drug delivery and live imaging strategies $-\mathrm{pH}$ responsive drug release [6], tumour oxygen and hypoxic sensors [7], locked nucleic and chimeric aptamer conjugates [8],fluorescence and bioluminescence approaches [9], ribonucleic acid interference (RNAi) technology [10], antibodies [11], nanobodies [12] magnetic guided therapy [13], sonophoto dynamic therapy (SPDT) - ultrasound and infrared laser [14], photodynamic therapy (PDT) [15], photothermal therapy (PTT) [15], radio imaging and therapy(RIT) [14], photoacoustic imaging(PAI) [16], magnetic resonance imaging and ultrasound therapy (MRI + US) [17], positron emission tomography(PET) [18], single-photon emission computed tomography (SPECT) [18].In turn, variant therapeutic abilities of SPIONS congregate to offer interactive therapeutic feedback monitoring over a time period, opening new vistas for personalised cancer nanomedicine [19].
Having a closer look at the conventional treatment regimes including chemotherapy, immunotherapy, it is a poignant fact that, these platforms certainly lack imaging ability, disqualifying them from being a theranostic [3]. Furthermore, existing radio therapeutic platforms that fall in basket of current theranostic medicine are extremely toxic and even trigger recurrent cancers leading to drug resistance [20]. This is where; SPIONS are conquering the therapeutic ground exhibiting versatility in derivatizing combinational drug systems - both with chemotherapeutic and natural product derived compounds (neem, curcumin, milk derived proteins) [21]. SPIONS are well known for their exceptional superparamagnetic ability with high relaxivity, outstanding bio degradability, targeted delivery, moreover can be easily integrated with a myriad of drug delivery platforms, achieving multimodal action [22]. Hence, SPIONS are extensively utilised as clinical imaging agents for magnetic resonance imaging [23]. Novel class of SPIONS derived MRI contrast agents include polyethylene glycol (PEG) - cleaved iron oxide nanoparticles (PEG - CIONPs)[23], magnetoliposomes [24], transferrin-conjugated SPIONS [22]. The existing class of MRI contrast agents such as gadolinium and manganese are highly toxic, exhibit nonspecific distribution in body, non biodegradable, cause extensive side effects and act as imaging only agent, lacking treatment ability [25].Inaddition to this, SPIONS also possess remarkable photoacoustic imagingability in response to the ultrasound frequencies that can be integrated with its magnetic property making it a dual imaging contrast agent [16]. Furthermore, both the ultrasound and magnetic property of SPIONS is widely exploited in targeted, controlled drug delivery and imaging applications [26]. Few of recent SPIONS based dual contrast imaging agents includepolyethylene glycol - doxorubicin - SPIONS (PEG - DOX - SPIONS) [26], endorem [27], magnetic microbubbles [17]. The existent ultrasound contrast agents such as optison, definity, albunex, Sonovue, based microbubble systems have a potential toxic effect, shorter half life [28] and also lack inherent therapeutic ability as compared to SPIONS derived nanotheranostics [26].

In conclusion, the holistic religion of SPIONS in the era of modern medical oncology offers infinite, exhilarating possibilities in targeted hurling of tumours, thereby quenching the thirst of cancer researchers and retaining the gist of healthy human kind in the cosmos [29-31].

\section{References}

[1]. Kanwar, R.K., Chaudhary, R., Tsuzuki, T. \& Kanwar, J.R. Emerging engineered magnetic nanoparticulate probes for molecular MRI of atherosclerosis: how far have we come? Nanomedicine7, 899-916 (2012).

[2]. Cheon, J. \& Lee, J.-H. Synergistically Integrated Nanoparticles as Multi- 
modal Probes for Nanobiotechnology. Accounts of Chemical Research41, $1630-1640$ (2008)

[3]. Svenson, S. Theranostics: Are We There Yet? Molecular Pharmaceutics (2013).

[4]. Kanwar, R.K., Chaudhary, R., Tsuzuki, T. \& Kanwar, J.R. Emerging engineered magnetic nanoparticulate probes for targeted MRI of atherosclerotic plaque macrophages. Nanomedicine7, 735-749 (2012).

[5]. Kim, D., Lee, J.-R., Shen, E. \& Wang, S. Modeling and experiments of magneto-nanosensors for diagnostics of radiation exposure and cancer. Biomed Microdevices, 1-7 (2012).

[6]. Diou, O., Tsapis, N. \& Fattal, E. Targeted nanotheranostics for personalized cancer therapy. Expert Opinion on Drug Delivery9, 1475-1487 (2012).

[7]. De Leon-Rodriguez, L.M., et al. Responsive MRI Agents for Sensing Metabolism in Vivo. Accounts of Chemical Research42, 948-957 (2009).

[8]. Kanwar, J.R., Roy, K. \& Kanwar, R.K. Chimeric aptamers in cancer celltargeted drug delivery. Critical Reviews in Biochemistry and Molecular Biology46, 459-477 (2011).

[9]. Nair, S.B., Dileep, A. \& Rajanikant, G.K. Nanotechnology Based Diagnostic and Therapeutic Strategies for Neuroscience with Special Emphasis on Ischemic Stroke. Current Medicinal Chemistry19, $744-756$ (2012).

[10]. Shim, M.S. \& Kwon, Y.J. Stimuli-responsive polymers and nanomaterials for gene delivery and imaging applications. Advanced Drug Delivery Reviews64, 1046-1059 (2012).

[11]. Kievit, F.M., et al. Targeting of Primary Breast Cancers and Metastases in a Transgenic Mouse Model Using Rationally Designed Multifunctional SPIONs. ACS Nano6, 2591-2601 (2012).

[12]. Sawant, R.R., Jhaveri, A.M. \& Torchilin, V.P. Immunomicelles for advancing personalized therapy. Advanced Drug Delivery Reviews64, 1436-1446 (2012).

[13]. Kim, D.-H., et al. Stimuli-responsive magnetic nanomicelles as multifunctional heat and cargo delivery vehicles. Langmuir (2013).

[14]. Li, A., et al. Superparamagnetic perfluorooctylbromide nanoparticles as a multimodal contrast agent for US, MR, and CT imaging. Acta Radiologica (2013).

[15]. Truby, R.L., Emelianov, S. \& Homan, K.A. Ligand-Mediated Self-Assembly of Hybrid Plasmonic and Superparamagnetic Nanostructures. Langmuir (2013).

[16]. Cook, J.R., Frey, W. \& Emelianov, S. Quantitative Photoacoustic Imaging of Nanoparticles in Cells and Tissues. ACS Nano (2013).

[17]. Owen, J., Pankhurst, Q. \& Stride, E. Magnetic targeting and ultrasound mediated drug delivery: Benefits, limitations and combination. International Journal of Hyperthermia28, 362-373 (2012).
[18]. Amstad, E., et al. Surface Functionalization of Single Superparamagnetic Iron Oxide Nanoparticles for Targeted Magnetic Resonance Imaging. Small5, 1334-1342 (2009).

[19]. Kircher, M.F., Mahmood, U., King, R.S., Weissleder, R. \& Josephson, L. A Multimodal Nanoparticle for Preoperative Magnetic Resonance Imaging and Intraoperative Optical Brain Tumor Delineation. Cancer Research63, 8122-8125 (2003).

[20]. Kanwar, J.R., Singh, N. \& Kanwar, R.K. Role of nanomedicine in reversing drug resistance mediated by ATP binding cassette transporters and Pglycoprotein in melanoma. Nanomedicine6, 701-714 (2011).

[21]. Kanwar, J.R., Kamalapuram, S.K. \& Kanwar, R.K. Survivin Signaling in Clinical Oncology: A Multifaceted Dragon. Medicinal Research Reviews, n/a-n/a (2012).

[22]. Jiang, W., et al. Conjugation of Functionalized SPIONs with Transferrin for Targeting and Imaging Brain Glial Tumors in Rat Model. PLoS ONE7, e37376 (2012)

[23]. Jeon, S.L., Chae, M.K., Jang, E.J. \& Lee, C. Cleaved Iron Oxide Nanoparticles as T2 Contrast Agents for Magnetic Resonance Imaging. Chemistry - A European Journal, n/a-n/a (2013).

[24]. Lorenzato, C., et al. MRI contrast variation of thermosensitive magnetoliposomes triggered by focused ultrasound: a tool for image-guided local drug delivery. Contrast Media \& Molecular Imaging8, 185-192 (2013).

[25]. Hao, D., et al. MRI contrast agents: Basic chemistry and safety. Journal of Magnetic Resonance Imaging36, 1060-1071 (2012).

[26]. Niu, C., et al. Doxorubicin loaded superparamagnetic PLGA-iron oxide multifunctional microbubbles for dual-mode US/MR imaging and therapy of metastasis in lymph nodes. Biomaterials34, 2307-2317 (2013).

[27]. Grootendorst, D.J., et al. Evaluation of superparamagnetic iron oxide nanoparticles $\left(\right.$ Endorem $^{\circ}$ ) as a photoacoustic contrast agent for intra-operative nodal staging. Contrast Media \& Molecular Imaging8, 83-91 (2013).

[28]. Alzaraa, A., et al. Targeted microbubbles in the experimental and clinical setting. The American Journal of Surgery204, 355-366 (2012).

[29]. Kanwar, J.R., Kamalapuram, S.K. \& Kanwar, R.K. Targeting survivin in cancer: the cell-signalling perspective. Drug Discovery Today16, 485-494 (2011).

[30]. Kanwar, J.R., Kanwar, R.K., Mahidhara, G. \& Cheung, C.H.A. Cancer Targeted Nanoparticles Specifically Induce Apoptosis in Cancer Cells and Spare Normal Cells. Australian Journal of Chemistry65, 5-14 (2012).

[31]. Kanwar, J.R., Mahidhara, G. \& Kanwar, R.K. Novel alginate-enclosed chitosan-calcium phosphate-loaded iron-saturated bovine lactoferrin nanocarriers for oral delivery in colon cancer therapy. Nanomedicine7, 1521-1550 (2012). 